\title{
25-Gauge Vitrectomy with Air Tamponade and 12 Hours Prone Positioning for a Stage 4 Macular Hole
}

\author{
Andrei Merticariu', Ramona Barac ${ }^{1,2}$, Florian Balta ${ }^{1,2}$, Mihai Pop ${ }^{1,2}$ \\ Corresponding author: \\ Ramona Barac, MD \\ Clinical Emergency Eye Hospital \\ No. 1, Alexandru Lahovari Plaza \\ Bucharest, Romania \\ E-mail: ramona.barac@live.com \\ ${ }^{1}$ Clinical Emergency Eye Hospital, Bucharest, Romania \\ 2"Carol Davila" University of Medicine and Pharmacy, Bucharest, Romania
}

\section{ABSTRACT}

Background: Macular hole is a rare retinal pathology with complex treatment options. All the modern surgical procedures used can achieve a high percentage ofanatomical hole closure, but functional recovery is incomplete and vulnerable to multiple complications.

Case report: We present the case of a 61-year-old female with a history of high myopia, left eye high amblyopia, right eye operated retinal detachmentand cataract, diagnosed in 2012 with recent stage 4 macular hole on her single eye. We decided to perform in this case a nonstandard procedure: transconjunctival $25-\mathrm{G}$ vitrectomy with only anair endotamponade and 12 hours prone positioning.

Results: Despite the poor perspectives, the early postoperative outcome was favorable, without any significant complications and the final outcome was exceptionally good, with full visual function recovery and complete anatomical macular hole closure.

Conclusion: the fast resolution air tamponade and short postoperative patient positioning can be just as effective as the standard surgical procedure, can produce above-average visual function recovery and is particularly useful in single eye patients.

Abbreviations: 25-G - 25-Gauge, BCVA - best corrected visual acuity, ILM - internal limiting membrane, C3F8 - octafluoropropane, MH - macular hole, OCT - optical coherence tomography, SF6 - sulfur hexafluoride

Key words: 25-Gauge vitrectomy, short-term air tamponade and prone positioning, single eye macular hole

\section{INTRODUCTION}

The macular hole $(\mathrm{MH})$ is a complete defect of the neurosenzorial retina in the fovea and has a worldwide prevalence that varies between 0,02 and 0,17\%. It is diagnosed more common in females (the male - female ratio is $1: 2,4$ ) and usually after 60 years of age (1).

$\mathrm{MH}$ is an anatomical condition that benefits only from surgical treatment (vitrectomy). There is currently a wide variety of procedures used and although they are relatively equal as anatomical closure rates and functional outcome, 
there is a difference regarding patient compliance and early postoperative quality of life (2).

The most popular surgical method used in recent years consists of transconjunctival vitrectomy with internal limiting membrane peeling, expandable gas endotamponade and post-operative face-down (prone) positioning of the patient for a period of time between 24 hours and 7 days. This method has proven to be the most effective and can achieve $\mathrm{MH}$ closure rates of above $90 \%$ withsignificant visual acuity improvement: from a mean preopbest corrected visual acuity (BCVA) of 20/129 (median 20/100) to a mean postop BCVA of 20/36 (median 20/30) (3).

\section{CASE REPORT}

We present the case of a 61 year old female patient who was admitted in January 2012 in the Vitreo-retinal Surgery Department of the Bucharest Clinical Emergency Eye Hospital presenting a sudden decrease of vision in her single functional eye (right eye), starting three weeks prior to the admission. The patient history included a high myopia with anisometropia (-6 dioptres on her right eye and -16 dioptres on the left) and high amblyopia on her left eye. She underwent two prior surgical procedures in another clinic for regmatogenous retinal detachmentin April 2011 and for complicated cataract in October 2011.

The first surgical intervention performed the year before was the vitrectomy for a recent macula-on superior retinal detachment. The patient presented with a peripheric positive scotoma in the inferonasal visual field of her single eye, noticed two days before and was also diagnosed with incipient age-related cataract in both her eyes. The initial BCVA was 16/20 on her right eye and hand-movement in her left eye. She was addmited and underwent surgical treatment with local anesthesia on the same day. At that point, the initial surgeon successfully used endolaser and air tamponade to attach the retina and the early outcome was very good: the retina remained attached after the resolution of the gas bubble a week later and the BCVA maintained at 16/20. The only significant complication was the secondary developmentof a complicated cataract in her right eyein the following months.

The acceleration of the cataract formation following intraocular surgery is well documented, especially aftervitrectomy. The incidence of cataract is variable, reported from $6 \%$ up to $100 \%$ of cases, depending on the disease that prompted vitrectomy (4). Cataract typically develops after long/difficult vitrectomies, like for retinal detachment (especially when silicone oil endotamponade is used) and tend to develop later and slower for easier proceduressuch asepimacular membrane or macular hole surgery.

The clinical examinations performed in the next six months after surgery described the rapid evolution of the cataract and the slow decrease of the visual acuity, up to $10 / 20$ in the October 2011 check-up. This condition in her single-seeing-eye prompted the decision to surgically remove the complicated cataract. A second surgeon performed anextracapsular ultrasound phacoemulsification removal of the cataract and implantation of a posterior chamber intraocular acrylic hydrophobic lens. This procedure was done also with local retrobulbaranesthesia and the medical team described a quick recovery and very good visual outcome. The dioptric power of the intraocular lens was calculated keeping account of therefractive condition (high myopia of minus six dioptres) so the patient's postop refraction was approximately -2 dioptres, which allowed her to read without glasses and use spectacles only for far-vision. The BCVA in her right eye was 20/20 at the one-month postop ophthalmological check-up.

Three months after the second procedure, the patient was reffered to our Vitreo-retinal department for a sudden decrese of vision in her right eye. She described a central scotoma and metamorphopsia, noticed approximately 20 days before. BCVA was $5 / 20$ at this point, with normal intraocular pressure $(16 \mathrm{~mm} \mathrm{Hg})$ and the slit lamp biomicroscopy examination showed the well positioned artificial lens, without other anterior pole pathology. The fundus examination was performed with the help of a biomicroscope and an indirect ophthalmoscope and revealed a macular hole with attached retina. The optical coherence tomography OCT exam showed the full thickness retinal hole at the fovea and the posterior vitreous detachment above the macula. The OCT also provided the measurements of the $\mathrm{MH}$ (the diameter was over 400 microns at that time), confirming the final diagnosis of stage 4 macular hole and the need for surgical treatment. (fig. 1)

We decided to shadule the vitrectomy as soon as possible, keeping account that is was a sigle eye and that the succes rate lowers if there is a longer period of time between the $\mathrm{MH}$ onset and surgery. The patient underwent 25 gauge transconjunctival pars plana vitrectomy under local anesthesia. We planned and managed to remove the thickened internal limiting membrane (ILM) which contributed to the tangential traction on the retinal layers and eventually produced the retinal hole. We used Triamcinolone acetonide to colour/ mark the ILM and to facilitate the peeling of the membrane and, since the surgery was uneventfull up to 


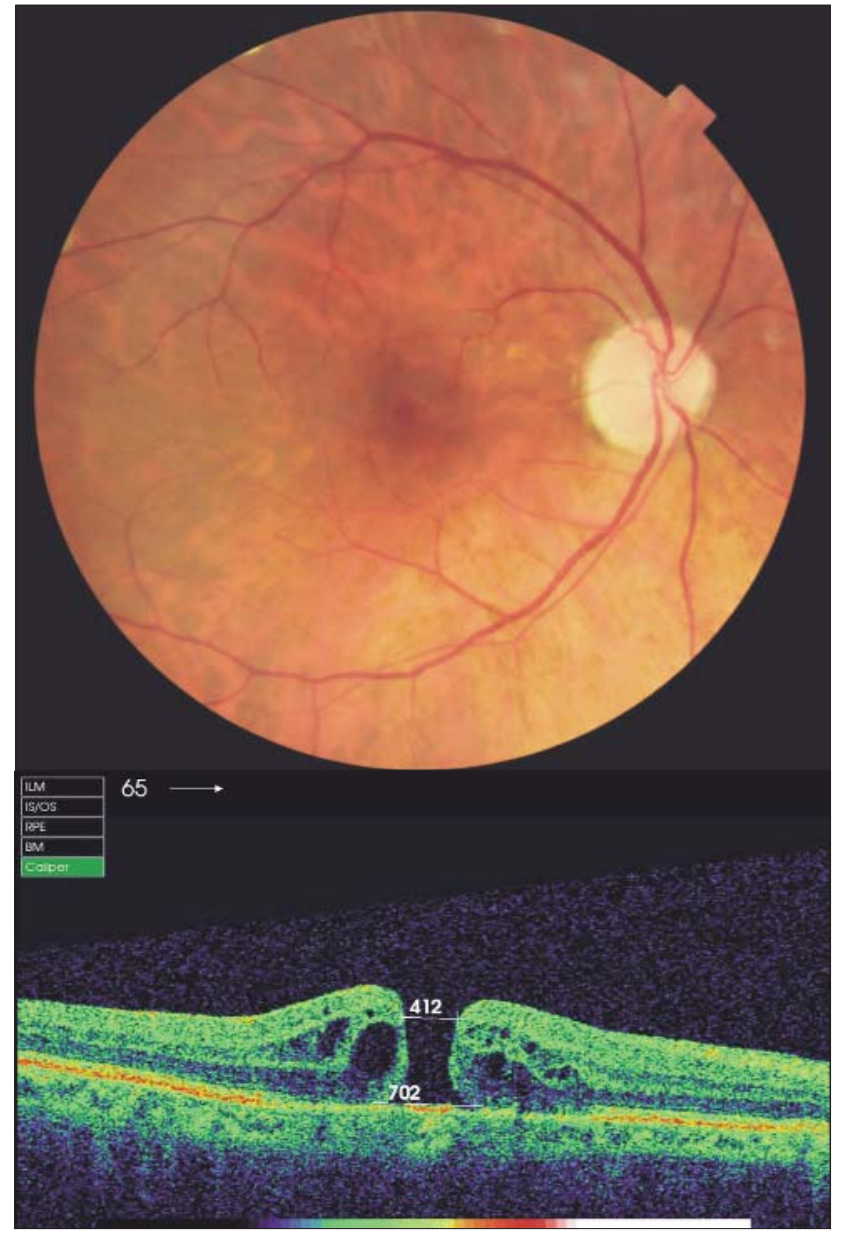

Figure 1 - Stage 4 macular hole (fundus image and OCT)

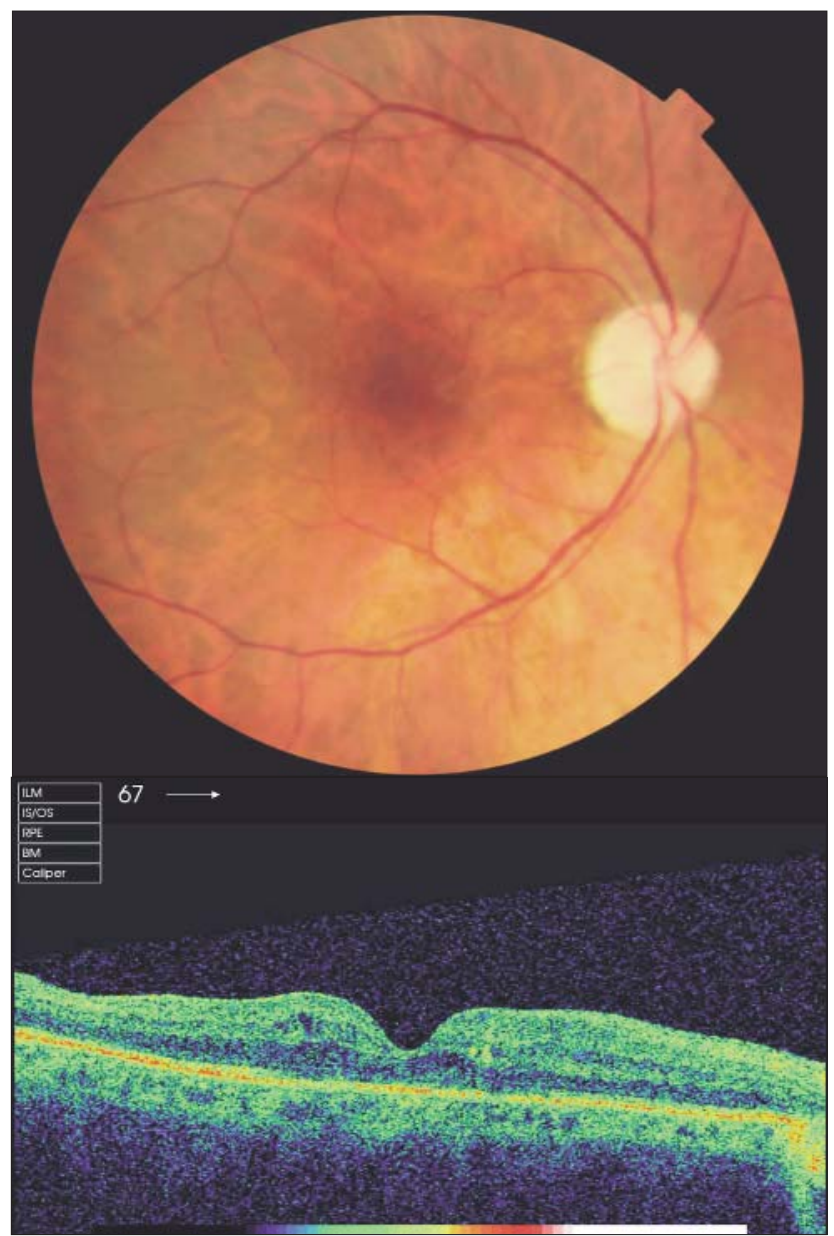

Figure 2 - Postoperative fundus image and OCT that point, we also decided to use only air for the endotamponade at the end of the surgery and avoid the use of long-lasting expandable gases. As with all $\mathrm{MH}$ surgical cases, we instructed the patient to mantain an overnight face-down prone postop position, so that the air bubble can apply pressure on the macular hole edges and keep it closed (5).

The second day check-up revealed a perfectly closed macular hole without any singnifficant complications and we didn't recommend to the patient any further prone positioning. The patient was discharged and was scheduled for a one week and a one month check-up. On both examinations, the OCT showed a perfectly closed $\mathrm{MH}$ and by this time the gas bubble absorbed completely. The patients BCVA had improved to 18/20 by the first week and $20 / 20$ by the first month postop. The outcome of this case was favorable and no late complications were described on the subsequent examinations. (fig. 2)

\section{DISCUSSION}

Macular hole formation is a very rare and severe complication after retinal detachment surgery, with an incidence of $0.24 \%$ (6). It carries a poor visual prognosis because it drasticly reduces the chances of visual acuity recovery. Although vitrectomy is the only curative treatment for $\mathrm{MH}$, there are currently several surgical variations available for this procedure with similar succes rates, but several differences in the reported complications.

25-Gauge vitrectomy has become the gold-standard in $\mathrm{MH}$ surgery, because of the easy-to-use transconjunctival trocars and the superior healing time and quality of life for this sutureless technique (less risk of wound leakage and infection, better comfort from the self-sealing sclerotomies). The most important surgical step in the peeling of the ILM to remove the tractional tension that ruptured the retina and to allow the closing of the retinal hole. However, from this point on, 
there are multiple options regarding surgical protocol that can be used. The "almost standard” procedure includes the use of expandable gas endotamponade at the end of the surgery and long term face down patient positioning to apply maximum pressure on the retina. With this protocol, surgical/anatomical succes rates can reach over $92 \%$ and functional recovery also improves significantly (7).

These gases, such as C3F8 or SF6, are most frequently used in macular hole surgery, because over the next few days thebubble will increase in size and apply more intraocular pressure to keep the retina attached, or in this case, to keep the macular hole closed for a longer period of time. Depending of the type of expandable gas used and the dilution chosen, this tamponade can be maintained inside the eye for up to four weeks, thus increasing the chances of $\mathrm{MH}$ closure. $(8,9,10)$ The expandable gases imply also a few disadvantages/ complications. First of all, the visual acuity is almost absent (hand-movement) while the gas bubble is inside the vitreous cavity, and second, there is a high risk of secondary glaucoma if the bubble expands more than anticipated level and the intraocular pressure rises. Air is sometimes used as an alternative because of several advantages: it has a shorter absorbtion time of around 5-7 days and it cheaper (free). It produces a shorter/ weaker tamponade ant it is recommended only in less-severe cases or pathologies.

There are also several options regarding early postoperative medical care. The patient prone positioning is very important because it keeps the gas bubble in constant contact with the macula. In the first days after surgery, the vitreal cavity will slowly start to fill up with fluid (aqueoushumour) and the gas bubble will move around depending of gravity (in an antigravitational manner). To keep constant pressure on the macular hole edges, most surgeons recommend mantaining a long term prone position, up to seven days. This step usually implies a prolonged hospitalisation time and higher cost of the inervention, and also a low patient compliance with poor quality of life in the first week postoperative $(11,12)$.

In our particular case, we were limited in our options by the fact that the disease occurred in a single functional eye and that the use of long acting expandable gases would prevent our patient from resuming her day-to-day activities and would induce a poor patient compliance, without adding any significantly superior closure rates. Recent studies have shown that air and SF6 are noninferior to long-lasting gases in the surgical management of $\mathrm{MH}$ (11). This patient already underwent a succesful air tamponade vitrec- tomy in the previous year, so we decided to use a short-acting air bubble for endotamponade and overnight prone positioning.

The next day fundus examination confirmed the complete closure of the macular hole, which allowed us to stop the patient positioning and continue monitoring the eye. The air bubble was quickly absorbed and reduced by half over the next four days, by which time the patient recovered half of her visual field and became able to partially resume her usual activities. At the seven day check-up the air absorbed completely, visual acuity improved to $18 / 20$ and by four weeks we noticed a full recovery to 20/20 vision. This was in fact the particularity of this case, because $20 / 20$ vision is a target very hard to reach in vitreo-retinal surgery, even with theese modern techniques and especially in cases with multiple surgery. Over the course of ten months, this single-eye patient was diagnosed with three different and potentially blinding conditions and underwent three successful different surgical interventions, with a well above-average final outcome, both anatomical and functional.

\section{CONCLUSIONS}

Simple air tamponade and short-term prone positioning are becoming increasingly popular in the management of macular hole treatment and are recently proven noninferior to the standard protocol. Their advantages are also of particullar importance in selected single-eye patients, with lower hospitalisation time and costs, better patient compliance and superior quality of life in the first month postoperative.

\section{Conflict of interests}

There is no conflict of interests.

\section{Acknowledgement}

This paper is financially supported by the project "CERO - PROFIL DE CARIERĂ: CERCETĂTOR ROMÂN", under the contract number POSDRU/ 159/1.5/ S / 135760, project co-financed from the European Social Fund by the Sectoral Operational Programme "Human Resources Development 2007-2013".

\section{REFERENCES}

1. McCannel CA, Ensminger JL, Diehl NN, Hodge DN. Population-based incidence of macular holes. Ophthalmology. 2009 Jul;116(7):1366-9.

2. Merticariu A, Balta F, Pop M. Macular hole treatment. J. Transl. Med. Res. 2015 Dec; 20(4):195-200. 
3. Scott IU, Moraczewski AL, Smiddy WE, Flynn HW Jr, Feuer WJ. Longterm anatomic and visual acuity outcomes after initial anatomic success with macular hole surgery. Am J Ophthalmol. 2003 May; 135(5): 633-40.

4. Do DV, Gichuhi S, Vedula SS, Hawkins BS. Surgery for postvitrectomy cataract. Cochrane Database Syst Rev. 2013 Dec 19; (12):CD006366.

5. Rizzo S, Belting C, Cresti F, Genovesi-Ebert F. Sutureless 25-gauge vitrectomy for idiopathic macular hole repair. Graefes Arch Clin Exp Ophthalmol. 2007 Oct;245(10):1437-40.

6. Lee SH, Park KH, Kim JH, Heo JW, Yu HG, Yu YS, et al. Secondary macular hole formation after vitrectomy. Retina. 2010 Jul-Aug;30(7): 1072-7

7. Veith $M$, Straňák Z, Penčák $M$, Studený $P$. Surgical Treatment of the Idiopathic Macular Hole by Means of 25-Gauge Pars Plana Vitrectomy with the Peeling of the Internal Limiting Membrane Assisted by Brilliant Blue and Gas Tamponade. Cesk Slov Oftalmol. 2015 Jun; 71(3):170-4. Czech
8. Garcia-Arumi J, Correa CA, Corcostegui B. Comparative study of different techniques of intraocular gas tamponade in the treatment of retinal detachment due to macular hole. Ophthalmologica. 1990; 201(2):83-91.

9. Carvounis PE, Kopel AC, Kuhl DP, Heffez J, Pepple K, Holz ER. 25gauge vitrectomy using sulfur hexafluoride and no prone positioning for repair of macular holes. Retina. 2008 0ct;28(9):1188-92.

10. Rahman R, Madgula I, Khan K. Outcomes of sulfur hexafluoride (SF6) versus perfluoroethane (C2F6) gas tamponade for non-posturing macular-hole surgery. Br J Ophthalmol. 2012 Feb;96(2):185-8.

11. Essex RW, Kingston ZS, Moreno-Betancur M, Shadbolt B, Hunyor AP, Campbell WG, et al. The Effect of Postoperative Face-Down Positioning and of Long-versus Short-Acting Gas in Macular Hole Surgery: Results of a Registry-Based Study. Ophthalmology. 2016 May;123(5):1129-36

12. Tatham A, Banerjee S. Face-down posturing after macular hole surgery: a meta-analysis. Br J Ophthalmol. 2010 May;94(5):62631. 